\title{
La carte des lieux. Connaissance et représentation de la géographie vernaculaire en breton
}

The map of places: knowledge and representation of vernacular geography in Breton

Nelly Blanchard et Mannaig Thomas

\section{OpenEdition}

\section{Journals}

Édition électronique

URL : http://journals.openedition.org//bl/440

DOI : $10.4000 / \mathrm{lbl} .440$

ISSN : 2727-9383

\section{Éditeur}

Université de Bretagne Occidentale - UBO

\section{Édition imprimée}

Date de publication : 1 mars 2016

Pagination : 161-180

ISBN : 979-10-92331-24-0

ISSN : $1270-2412$

\section{Référence électronique}

Nelly Blanchard et Mannaig Thomas, «La carte des lieux. Connaissance et représentation de la géographie vernaculaire en breton », La Bretagne Linguistique [En ligne], 20 | 2016, mis en ligne le 01 mai 2020, consulté le 01 octobre 2020. URL : http://journals.openedition.org//bl/440 ; DOI : https:// doi.org/10.4000/lbl.440

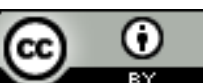

La Bretagne Linguistique est mise à disposition selon les termes de la Licence Creative Commons Attribution 4.0 International. 
Nelly BLANCHARD* et Mannaig THOMAS*

\title{
La carte des lieux. Connaissance et représentation de la géographie vernaculaire en breton
}

\begin{abstract}
L
a carte des lieux qui sera dressée dans cet article rendra visible la connaissance et la représentation de l'espace, en tant que savoir vernaculaire, et exprimé en breton. C'est donc bien de géographie vernaculaire, au sens de savoir géographique de sens commun - distinct du savoir des géographes - dont il sera question. Les données de cette enquête ont été collectées récemment auprès de bretonnants dont le breton est la langue première comme langue socialement héritée. Les deux volets que sont la connaissance et la représentation de l'espace seront ici abordés, autrement dit la capacité à nommer, à se situer, mais également la manière de percevoir l'environnement géographique. Comme la perception de l'environnement est filtrée par l'expérience, par l'attitude de chacun vis-à-vis de cet environnement et par les comportements adoptés en son sein, et qu'elle est par ailleurs influencée par l'évocation de lieux mentaux, vont apparaître ici des espaces vécus, des espaces perçus et des espaces projetés.

Après un $\mathrm{XX}^{\mathrm{e}}$ siècle qui a grandement et brusquement élargi les possibles horizons géographiques et mentaux des individus, et un $\mathrm{XXI}^{\mathrm{e}}$ siècle qui voit la promotion de l'image de Bretons « enracinés et ouverts sur le monde ${ }^{1} »$, nous cherchons à percevoir et comprendre l'image correspon-

* Maîtres de conférences de breton et celtique, CRBC (EA 4451), UBO.

1. Expression très fréquemment employée par le personnel politique et médiatique au niveau régional. Voir par exemple Jean-Michel LE BoulANGER, Etre breton?, Quimper, Palantines, 2013.
\end{abstract}


dant à la carte des lieux actuelle des enquêtés, par le vecteur linguistique breton, au début du XXI ${ }^{\mathrm{e}}$ siècle. En d'autres termes, comment ce siècle d'« ouverture sur le monde » se manifeste-t-il dans ce savoir vernaculaire breton?

\section{Protocole d'enquête : Brezhoneg war an dachenn}

Brezhoneg war an dachenn est un projet mené par Mannaig Thomas et Nelly Blanchard dans le cadre d'un cours de Master 1 recherche ${ }^{2}$ visant, d'une part, à familiariser les étudiants à l'enquête de terrain, et d'autre part, à réaliser collectivement et sur plusieurs années une collecte de données en dialectologie et sociolinguistique bretonnes.

Premièrement, l'objectif pédagogique est de mettre sur pied de véritables enquêtes de terrain comprenant la préparation (prise de contact, logistique, questionnaires...), la réalisation (déplacements, enregistrements, confection de notices de référencement, gestion du volet juridique de l'enquête, etc.) et la restitution des résultats (sur format papier, audio ou autre), tout en exploitant les méthodes acquises en licence dans les domaines concernés. Deuxièmement, il s'agit, dans le cadre de cette enquête à grande échelle reposant sur un questionnaire commun, de collecter, d'archiver et d'exploiter des données dialectales et sociolinguistiques dans le domaine breton, l'objectif étant à terme de couvrir l'ensemble du territoire bas-breton. L'archivage est assuré par le Centre de recherche bretonne et celtique où les enregistrements pourront être consultés.

L'enquête de dialectologie s'appuie sur deux grands projets qui n'ont pas pu voir le jour jusqu'à présent :

- le Nouvel Atlas Linguistique de Basse Bretagne 2 (NALBB2) consacré à la morphologie et à la syntaxe du breton vernaculaire ${ }^{3}$,

- le vocabulaire de la vie champêtre dont nous avons extrait les questions portant sur le thème de l'eau ${ }^{4}$.

Nous avons demandé également un enregistrement libre de trois minutes au minimum où chaque informateur s'exprime en continu.

2. M1 Identités, patrimoines, histoire, parcours celtique, Université de Bretagne Occidentale.

3. Ce projet, né en 1995, n'a pas abouti et a été relancé en 2005 par Jean Le Dû et Nelly Blanchard qui s'étaient entourés d'une équipe d'une vingtaine d'enquêteurs bénévoles. Plusieurs types de difficultés ont été rencontrés qui n'ont pas permis de mener le travail à son terme.

4. C'est également un projet conçu par Jean Le Dû au milieu des années 1990. Ce projet n'a fait l'objet que de la confection du questionnaire. Il comporte à la fois des mots, des phrases à traduire et des mots à nommer à partir d'images. 
L'enquête de sociolinguistique est, quant à elle, originale et part de la constatation que les questionnaires sociolinguistiques sont souvent quantitatifs et rarement qualitatifs. On cherche ici à explorer les différents domaines de la vie (parler d'amour, de la mort, de l'argent...) par le biais des pratiques linguistiques. Il s'agit de s'interroger sur la place respective qu'occupaient et qu'occupent encore le breton et le français dans l'usage quotidien des bretonnants, ainsi que comprendre comment s'est produit le passage progressif d'une langue à l'autre en fonction des locuteurs et des différentes situations d'énonciation. À l'exploration des sphères sociales et temporelles s'ajoute une très brève partie sur l'espace : il s'agit de mieux comprendre la représentation du territoire par la capacité à nommer en breton différentes communes ou villes.

C'est sur les réponses à cette toute petite partie de l'enquête que porte cet article.

\section{Bilan actuel de l'enquête}

L'enquête a démarré en 2011. Sur une base d'un informateur par canton, les étudiants ont pour le moment couvert 50 cantons (voir la carte en annexe), ce qui représente environ $60 \%$ des cantons de la BasseBretagne. Cela a mobilisé 30 étudiants ${ }^{5}$ et 64 informateurs bretonnants ${ }^{6}$. Concrètement, chaque année nous choisissons collectivement une ou deux zones resserrées à couvrir, à charge aux étudiants de trouver eux-mêmes leurs informateurs. De ce choix pédagogique découle l'une des spécificités de cette enquête : le seul critère qui rassemble l'ensemble des informateurs est qu'ils sont bretonnants de naissance. Nous avions évoqué d'autres critères qui sont classiquement retenus dans les enquêtes dialectologiques (personne originaire du lieu étudié, leurs parents également, informateurs ne sachant ni lire ni écrire le breton), mais force est de constater les limites de ce concept se frottant à la réalité sociolinguistique du breton du $\mathrm{XXI}^{\mathrm{e}}$ siècle : peut-être l'ensemble des étudiants ne sont-ils pas des enquêteurs idéaux, sans doute ne perçoivent-ils pas l'intérêt du profil de

5. Les îles ont été couvertes grâce à des vacations car les enquêtes étaient plus difficilement réalisables dans l'organisation des déplacements des groupes.

6. La différence entre le nombre de cantons couverts et le nombre d'informateurs vient du fait que certains cantons ont été couverts plusieurs fois (par exemple, l'île de Batz fait partie du canton de Saint-Pol-de-Léon, mais nous pensons qu'il fallait que l'île de Batz soit prise en compte pour elle-même, comme toutes les autres îles d'ailleurs) et du fait que certaines enquêtes ont été menées auprès de couples ou de plusieurs personnes à la fois. 
l'informateur idéal, peut-être aussi l'informateur idéal n'existe-t-il plus ou pas.

L'enquête présente donc la particularité de s'appuyer sur des informateurs aux profils très divers et des manières d'enquêter peu uniformes. Pour autant, nous avons collecté grâce à cette enquête beaucoup de données tout à fait exploitables.

\section{Résultats}

\section{Portrait sociologique des informateurs}

Les tableaux et graphiques ci-dessous exposent les caractéristiques d'âge, de sexe et de profession des 31 informateurs dont les réponses sont étudiées dans cet article.

Fig. 1 : Tableau de répartition des informateurs par âge et par sexe

\begin{tabular}{|l|c|c|c|}
\cline { 2 - 4 } \multicolumn{1}{c|}{} & Homme & Femme & Total \\
\hline Moins de 50 ans & 1 & 0 & $1(3,2 \%)$ \\
\hline $50-60$ ans & 2 & 0 & $2(6,4 \%)$ \\
\hline $60-70$ ans & 2 & 0 & $2(6,4 \%)$ \\
\hline $70-80$ ans & 4 & 5 & $9(29 \%)$ \\
\hline $80-90$ ans & 8 & 7 & $15(48,4 \%)$ \\
\hline Plus de 90 ans & 1 & 1 & $2(6,4 \%)$ \\
\hline Total & $18(58 \%)$ & $13(42 \%)$ & 31 \\
\hline
\end{tabular}

Fig. 2 : Représentation graphique de l'âge des informateurs

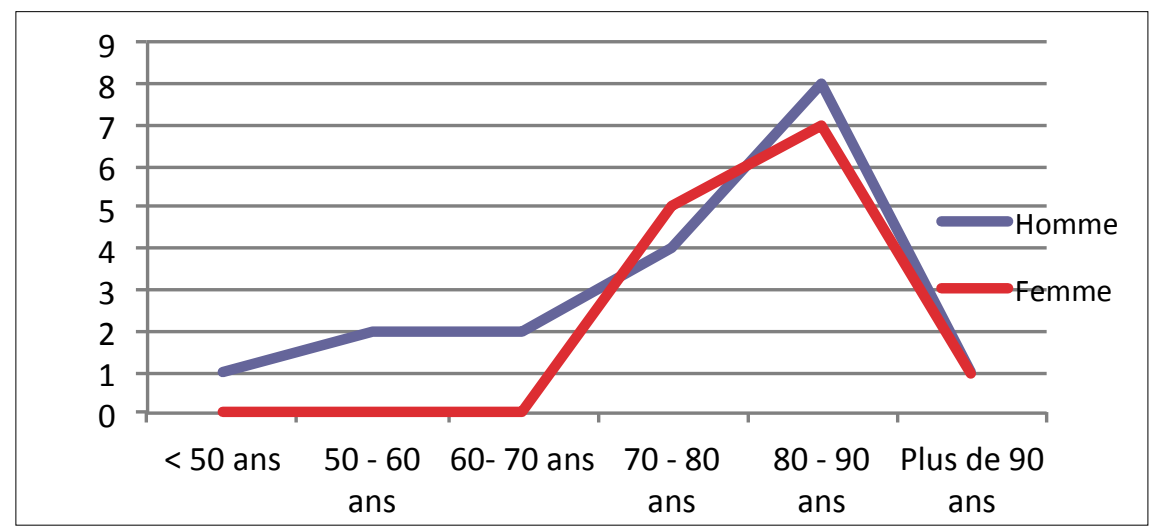


Fig. 3 : Tableau de répartition des informateurs par profession et par sexe

\begin{tabular}{|l|c|c|c|}
\cline { 2 - 4 } \multicolumn{1}{c|}{} & Homme & Femme & Total \\
\hline Agriculteur & 8 & 10 & $18(58 \%)$ \\
\hline Ouvrier & 3 & 0 & $3(9,7 \%)$ \\
\hline Employé bureau & 3 & 0 & $3(9,7 \%)$ \\
\hline Commerçant & 1 & 0 & $1(3,2 \%)$ \\
\hline Assistante maternelle & 0 & 1 & $1(3,2 \%)$ \\
\hline Agent de service EN & 1 & 0 & $1(3,2 \%)$ \\
\hline Chauffeur & 1 & 0 & $1(3,2 \%)$ \\
\hline Prêtre & 1 & 0 & $1(3,2 \%)$ \\
\hline Sans activité & 0 & 1 & $1(3,2 \%)$ \\
\hline Info. non connue & 0 & 1 & $1(3,2 \%)$ \\
\hline \multicolumn{1}{|c|}{ Total } & 18 & 13 & 31 \\
\hline
\end{tabular}

Fig. 4 : Représentation graphique de la profession des informateurs

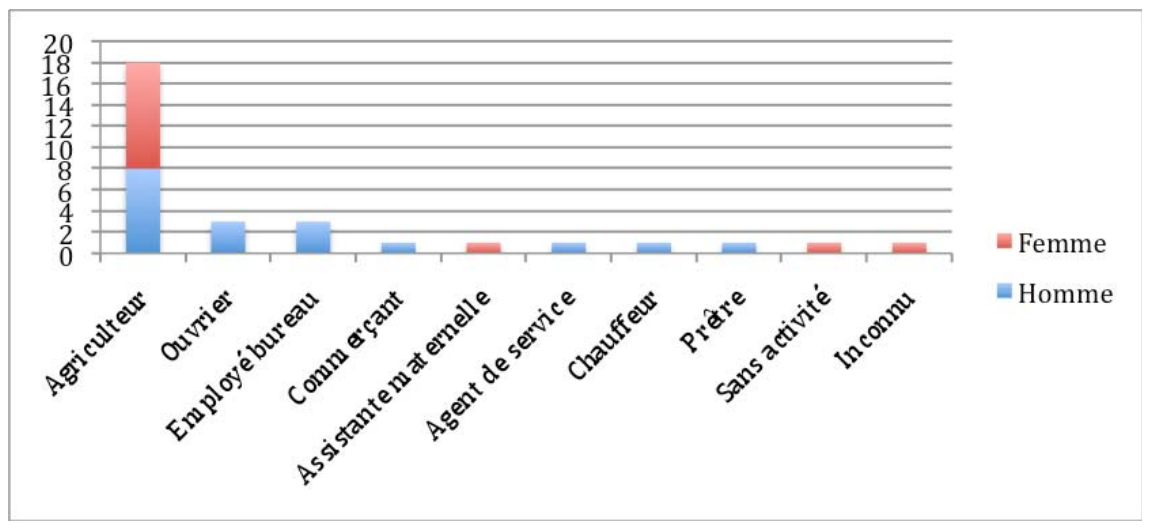

La carte (fig. 5) ${ }^{7}$ indique l'origine géographique des 31 informateurs étudiés, habitant 30 communes de Basse-Bretagne.

7. Nous avons réalisé l'ensemble des cartes de cet article avec l'aide d'Yves Guyomard que nous remercions vivement. 
Fig. 5 : Carte des communes d'origine des informateurs

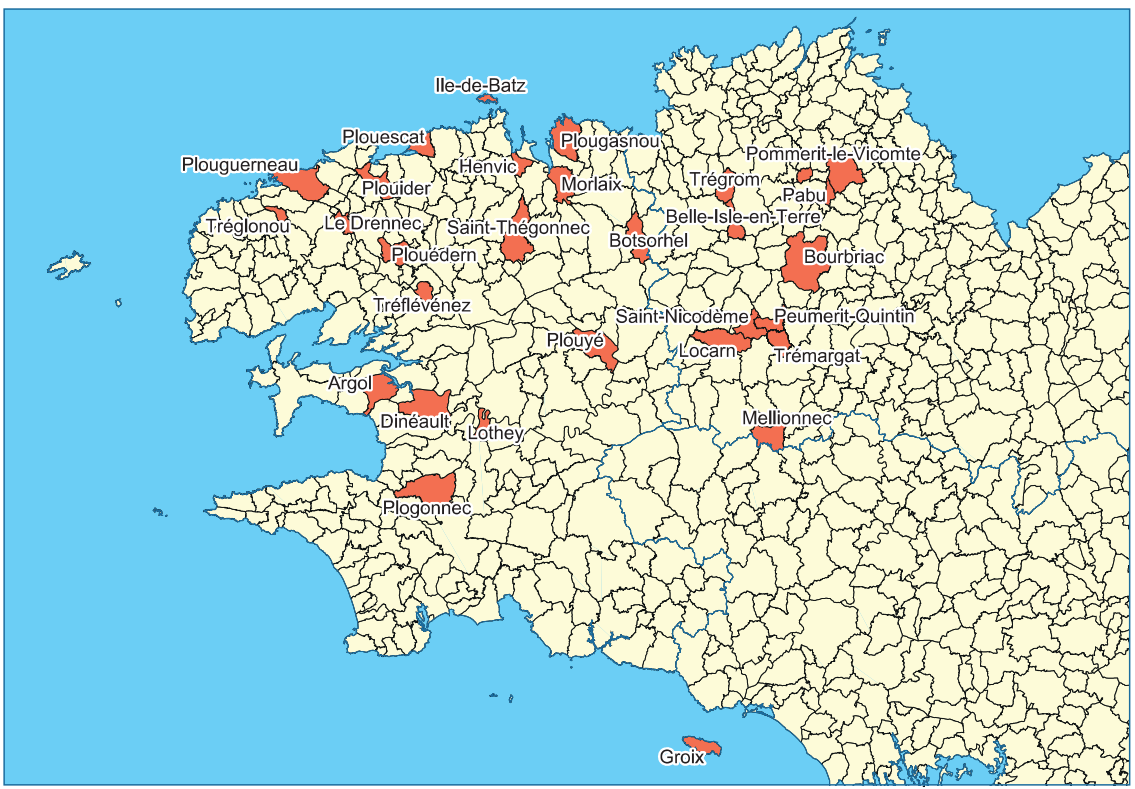

\section{Principales données chiffrées}

\section{Nommer des communes}

La manière de mener les enquêtes adoptée par les étudiants n'étant pas toujours uniforme, nous avons distingué les résultats obtenus à partir des réponses spontanées à la sollicitation générale de nommer des communes en breton, des réponses obtenues sur demande de traduction de noms de communes, et enfin d'autres propos libres concernant la géographie du territoire en question.

Les communes citées spontanément par les 31 informateurs sont en moyenne au nombre de 11,5. L'informateur en citant le moins n'en cite qu'une (Le Grouanec, qui d'ailleurs n'est pas une commune, mais une partie de la commune de Plouguerneau) et l'informateur qui en cite le plus en cite 37, se prenant au jeu des enquêteurs. Le plus souvent, ce sont 7 à 8 noms qui sont fournis. La carte (fig. 6) permet de visualiser les communes citées spontanément en breton par l'ensemble des informateurs.

La majorité des communes citées sont les communes voisines de la commune d'origine de l'informateur et représentent un espace géographique restreint. Le tableau suivant indique la répartition du nombre d'en- 
quêteurs par distance moyenne entre les communes citées et leur propre commune :

\begin{tabular}{|l|c|c|c|c|c|c|}
\hline Distance moyenne & $<10 \mathrm{~km}$ & $10-20 \mathrm{~km}$ & $20-30 \mathrm{~km}$ & $30-40 \mathrm{~km}$ & $40-100 \mathrm{~km}$ & $>100 \mathrm{~km}$ \\
\hline Nombre d'informateurs & 9 & 7 & 2 & 4 & 1 & 2 \\
\hline
\end{tabular}

$64 \%$ des informateurs citant spontanément des communes proposent une première image de leur espace mental distante de chez eux de moins de $20 \mathrm{~km} ; 88 \%$ ne dépassent pas $40 \mathrm{~km}$ autour de leur commune d'origine. La carte (fig. 7) illustre ce phénomène.

Fig. 6 : Carte des communes citées spontanément par les 31 informateurs

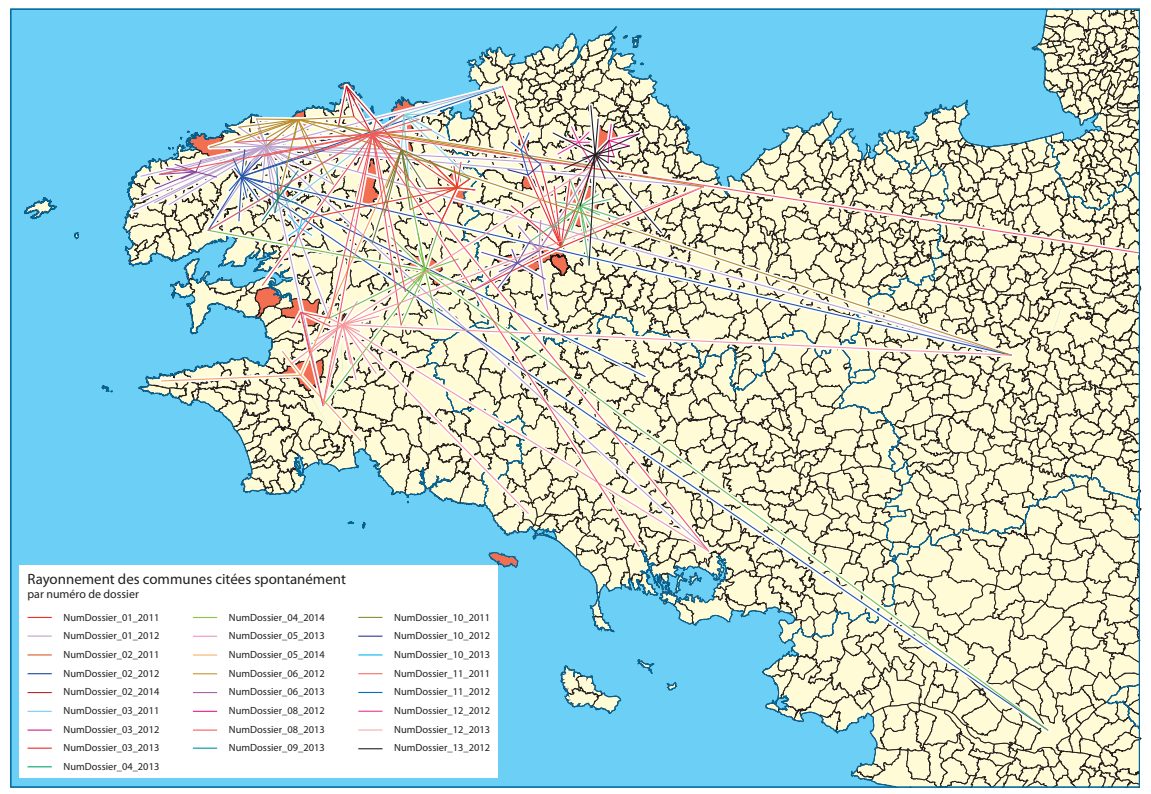

Si certains font minutieusement le tour des communes voisines, on constate chez d'autres informateurs des préférences spatiales qui les font prendre telle ou telle direction, comme l'informateur de Morlaix qui dit par ailleurs avoir été élevé dans les monts d'Arrée et qui cite naturellement un plus grand nombre de communes des monts d'Arrée; comme l'informatrice de Plougasnou qui manifeste une préférence pour la région de Morlaix et le Trégor finistérien, plus que pour le Trégor costarmoricain ; comme l'informatrice de Peumeurit-Quintin qui cite plus densément des communes situées au nord de chez elle et semble éviter le sud ; ou encore comme les deux informateurs de Botsorhel qui ne franchissent pas ou peu la frontière départementale finistérienne (fig. 8). 
Fig. 7 : Carte montrant quelques distances moyennes restreintes

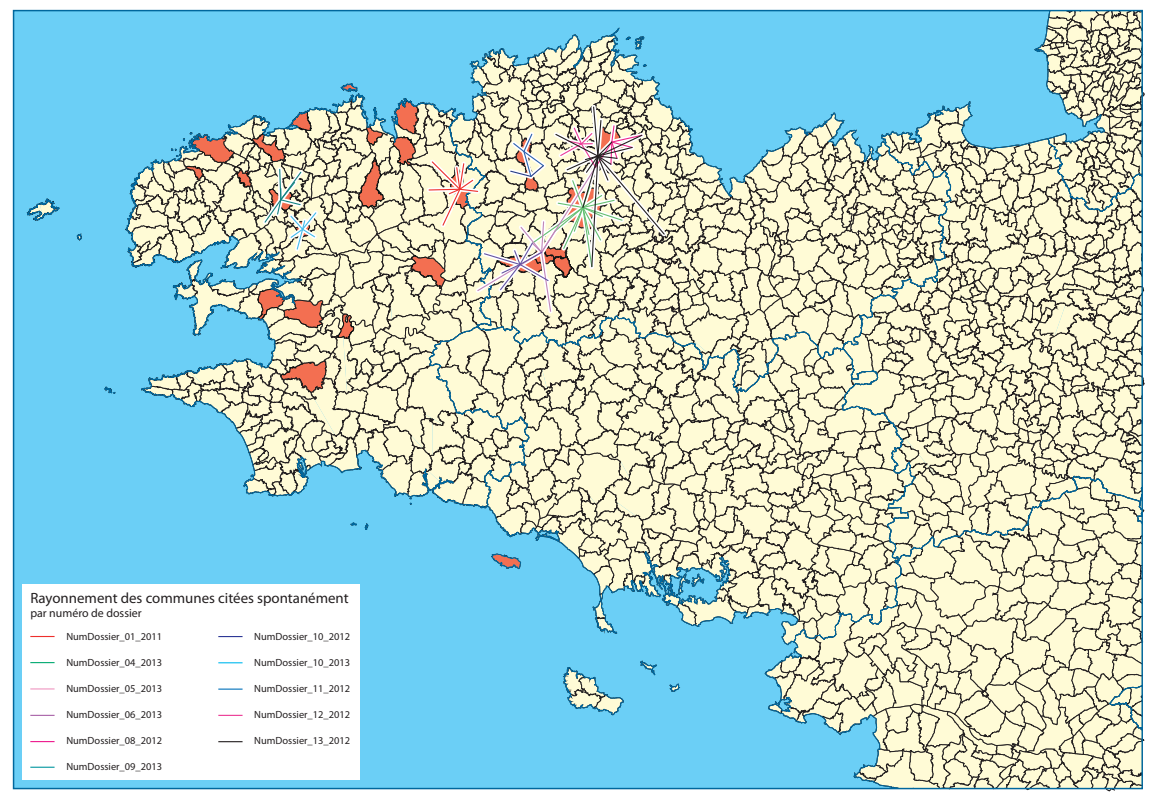

Fig. 8 : Cartes illustrant quelques préférences spatiales

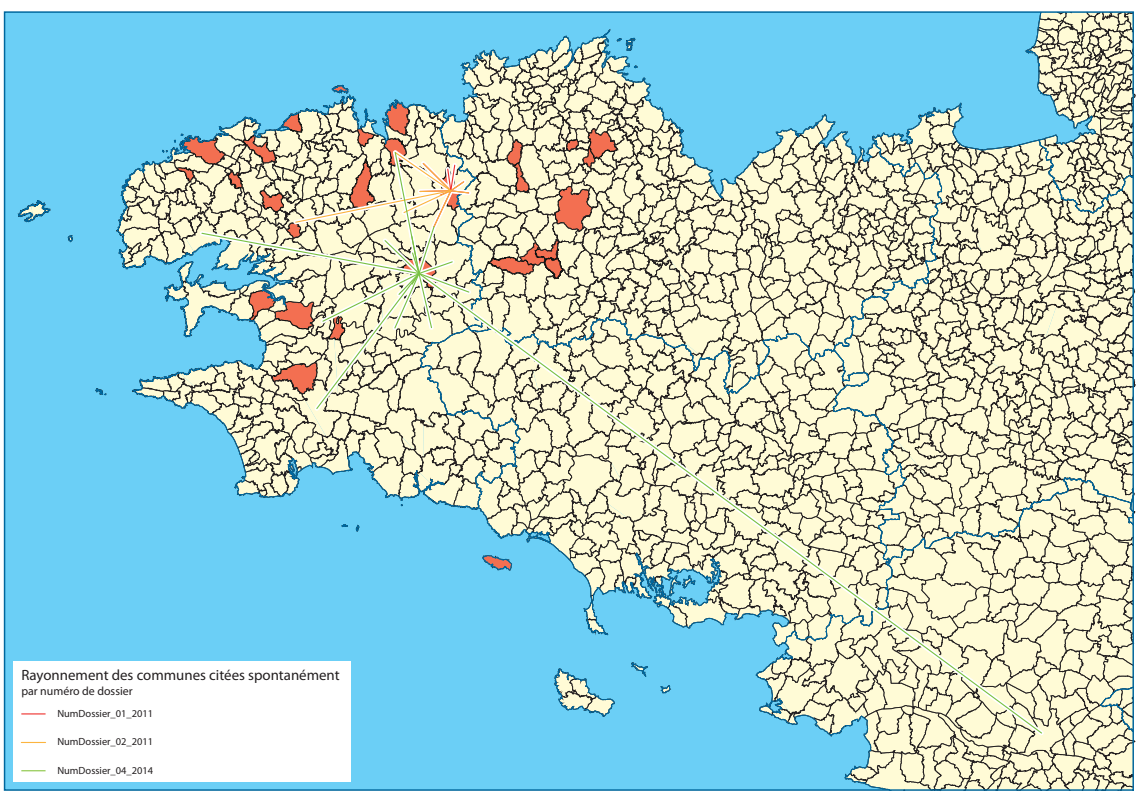


Pourtant quelques exceptions sont remarquables. Certains informateurs, bien que rares, dépassent largement à la fois le nombre de villes citées spontanément et la distance moyenne les séparant de ces villes citées. Les informateurs de Plouider et d'Henvic citent respectivement 37 et 27 communes spontanément, et couvrent ainsi des distances moyennes de $1200 \mathrm{~km}$ et $1050 \mathrm{~km}$ autour de chez eux (fig. 9).

Fig. 9 : Cartes de deux informateurs faisant exceptions
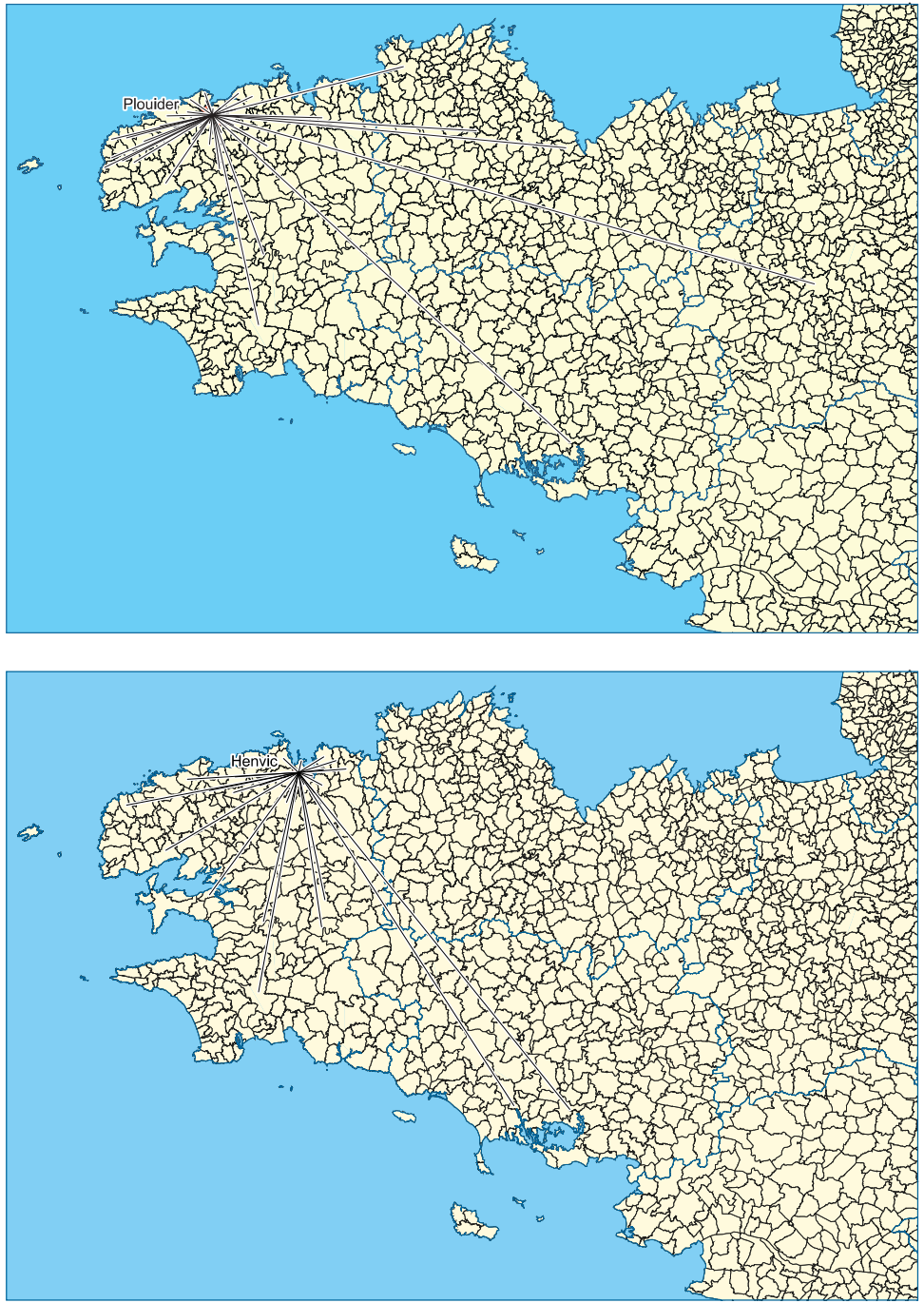
On peut noter que ces deux hommes sont les deux seuls employés de l'échantillon et, de manière plus générale, on note que ce sont les agriculteurs qui citent le plus petit nombre de communes et souvent les espaces les plus restreints de l'enquête. Cette donnée, vu l'échantillon étudié, croise celle du sexe : les femmes enquêtées citent moins de communes que les hommes. L'âge, en revanche, ne semble pas être un critère pertinent à ce sujet.

Fig. 10 : Tableau des correspondances entre les professions et le nombre de communes citées spontanément

\begin{tabular}{|l|c|}
\hline \multicolumn{1}{|c|}{ Profession de l'enquêté } & Nombre de communes citées \\
\hline employé de banque & 37 \\
employé & 27 \\
couvreur & 19 \\
militaire employé de banque & 16 \\
agent service EN & 16 \\
agriculteur & 15 \\
agriculteur & 15 \\
agriculteur & 14 \\
agriculteur & 11 \\
agriculteur & 9 \\
électromécanicien & 8 \\
agriculteur & 8 \\
commerçant & 8 \\
assistante maternelle & 7 \\
agriculteur & 7 \\
agricultrice & 7 \\
agriculteur & 7 \\
femme au foyer & 7 \\
agriculteur retraité & 7 \\
agriculteur & 6 \\
agriculteur & 5 \\
agriculteur & 5 \\
agriculteur & 5 \\
agriculteur & 0 \\
agriculteur maçon & 0 \\
retraité & 5 \\
agriculteur & 2 \\
agriculteur & 5 \\
\hline
\end{tabular}


Nous avons déjà eu l'occasion de signaler que les enquêtes n'ont pas toujours été menées de manière totalement identique. Certains enquêteurs ont préféré solliciter directement les informateurs pour des traductions et le faire dans le détail et de manière ordonnée, allant des communes les plus proches aux communes les plus éloignées. Cette procédure, qui donne des résultats différents de la citation spontanée, permet de repérer plus nettement les points de rupture dans la connaissance des formes bretonnes. Quand la méthode des réponses spontanées permet davantage d'approcher des préférences géographiques, cette deuxième méthode montre plus fidèlement la connaissance de la géographie en breton. Les zones alors couvertes sont plus larges, sans toutefois couvrir une superficie équivalente à un tiers de département ${ }^{8}$.

Fig. 11 : Cartes obtenues à partir de demandes de traductions uniquement
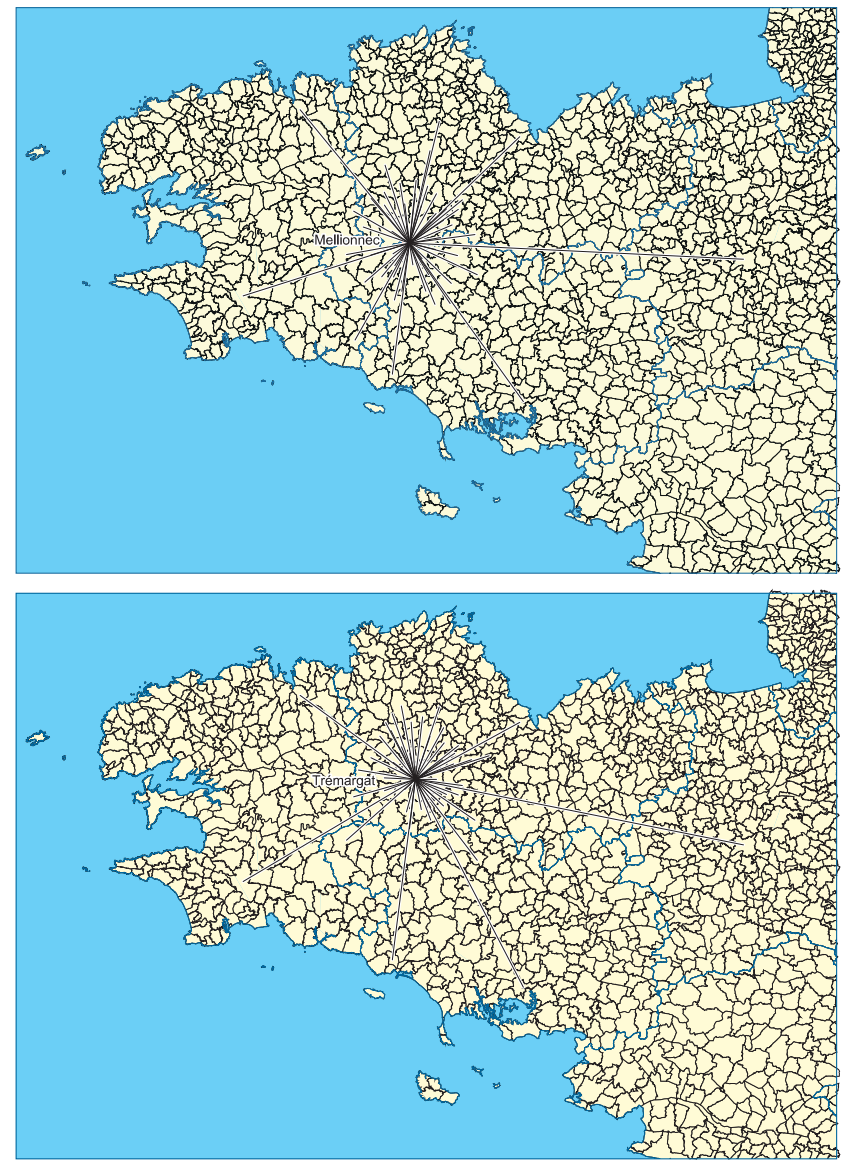

8. On peut noter par ailleurs que le cercle périphérique des dernières villes traduites est souvent plus approximatif et hypothétique. 
Certaines grandes villes sont également citées spontanément. Il s'agit alors, dans l'ordre décroissant, de Guingamp (10 informateurs sur 31), Morlaix (7/31), Brest (5/31), Quimper (5/31), Rennes (4/31), Saint-Brieuc (4/31), Vannes (3/31), Carhaix (3/31), Nantes (2/31), Lorient (1/31). La carte ci-dessous en montre l'importance relative :

Fig. 12 : Carte de l'importance relative des grandes villes bretonnes citées spontanément

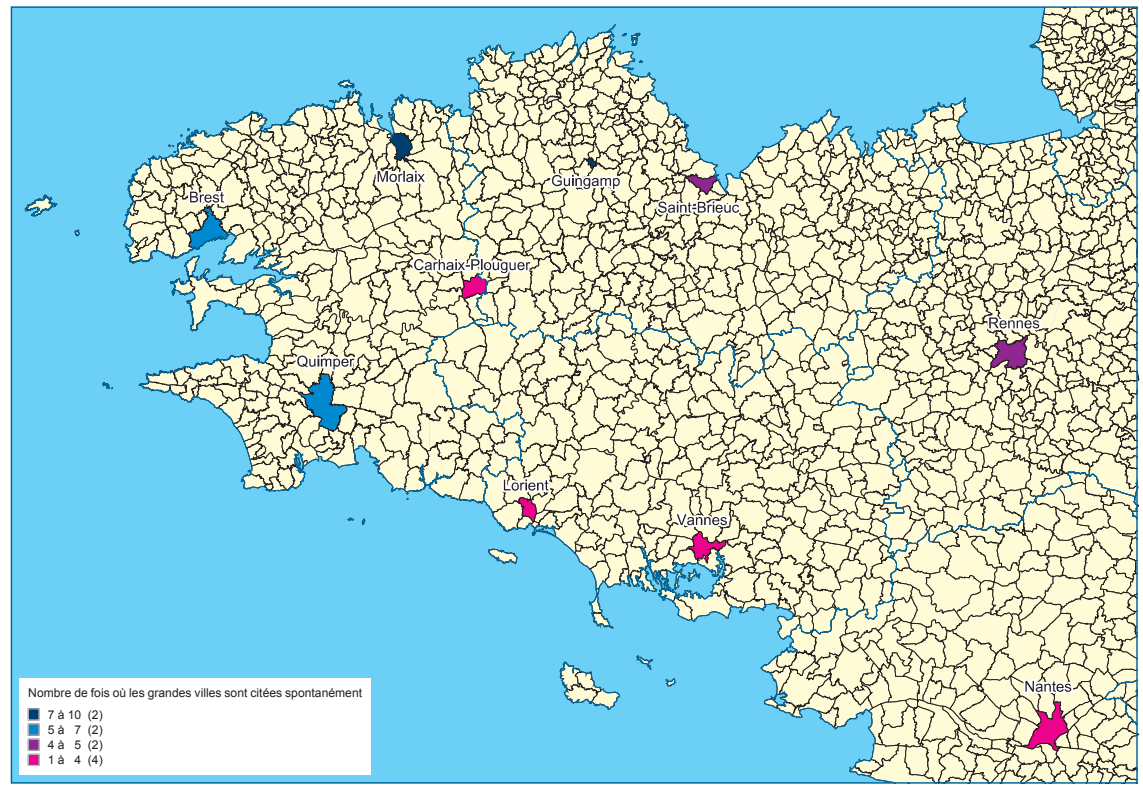

Le tableau ci-dessous fournit un classement des traductions des noms des principales communes obtenues sur sollicitations (ou déjà fournies spontanément). Pour compléter et nuancer ce tableau et cerner au mieux la maîtrise de cet espace mental en breton, il est intéressant de noter dans une troisième colonne les formes obtenues. On constate en effet une gradation entre les informateurs qui disent ne pas connaître une forme linguistique, ceux qui fournissent une réponse empruntée et calquée sur la forme française, ceux qui fournissent une réponse différente de la forme connue dans la commune en question, et enfin ceux qui fournissent la forme employée dans cette commune. 


\begin{tabular}{|l|l|l|}
\hline $\begin{array}{l}\text { Nom de la } \\
\text { commune }\end{array}$ & $\begin{array}{l}\text { Pourcentage } \\
\text { de réponses }\end{array}$ & Formes obtenues \\
\hline Morlaix & $100 \%(28 / 28)$ & Montroulez, Montourlez \\
\hline Carhaix-Plouguer & $100 \%(28 / 28)$ & Kerez, Karez, Karaez \\
\hline Rennes & $100 \%(28 / 28)$ & Roazon, Raon, Raouzoun, Renn $(1)$ \\
\hline Lorient & $100 \%(28 / 28)$ & Lorian $(16)$, An Oriant $(12)$ \\
\hline Quimper & $100 \%(26 / 26)$ & Keper, Keñper, Kemper \\
\hline Paris & $100 \%(25 / 25)$ & Pariz, Parich $(1)$ \\
\hline Guingamp & $100 \%(24 / 24)$ & Gwengam, Gwingam $(2)$ \\
\hline Brest & $100 \%(18 / 18)$ & Brest $(/ e /$ ou /e/) \\
\hline Saint-Brieuc & $96,5 \%(27 / 28)$ & Sant-Brieg, Zant-Brieg \\
\hline Vannes & $72,5 \%(21 / 29)$ & $\begin{array}{l}\text { Gwened, Gwenen }(1), \text { Vened }(1), \\
\text { Woned }(1), \text { Vann }(1)\end{array}$ \\
\hline Bordeaux & $35,7 \%(10 / 28)$ & Bourdel \\
\hline Le Havre & $35,7 \%(10 / 28)$ & An Havr $(7)$, Havr $(2)$, Hawr Nevez (1) \\
\hline
\end{tabular}

On constate que certaines formes ont peu de variantes, comme Morlaix, Carhaix, Quimper ou Paris, et d'autres en connaissent davantage, trahissant une moins bonne connaissance de la forme locale. Le contexte de l'interview explique en partie cette variété puisqu'il a poussé les informateurs à tâcher de fournir une réponse, parfois presque inventée à partir de l'emprunt français bretonnisé, ce qui transforme légèrement la perception des résultats. C'est le cas notamment de Lorient que $100 \%$ des informateurs ont traduit, mais dont seulement $43 \%$ fournissent la forme locale et $57 \%$ proposent la forme légèrement bretonnisée «Lorian ».

L'origine géographique des informateurs explique aussi en partie le fort taux de capacité de traduction des villes du nord et de l'ouest, à savoir Morlaix, Carhaix, Quimper. Le cas de Brest qui obtient peu de mentions vient du fait que l'ensemble des enquêteurs n'a pas sollicité les informateurs sur cette traduction; de plus, les réponses de certains informateurs montrent qu'ils ne trouvent pas toujours la question pertinente puisque les formes bretonne et française sont assez proches dans ce cas; on note enfin que ce sont surtout les informateurs du nord Finistère qui la citent spontanément, ce qui s'explique par la proximité géographique de la ville.

Paris et Rennes sont fortement traduits : les réponses assurées des informateurs montrent que ces villes portent des noms bretons et qu'ils sont connus. Paris ne connaît pour ainsi dire qu'une seule forme, alors que Rennes subit des transformations liées à des variantes dialectales 
(amuïssement du /z/, fermeture de la voyelle /o/ en /u/ devant $/ \mathrm{n} /$ en Léon, métathèse /aw/vs/wa/, etc.).

Enfin, on remarque que la connaissance des formes bretonnes d'une ville comme Bordeaux est tranchée : la forme est connue ou ne l'est pas, et elle est en l'occurrence connue par assez peu de personne. Le cas du Havre est un peu particulier : sa forme bretonne est très peu connue, mais pour autant, les informateurs proposent l'emprunt français bretonnisé (avec article breton et neutralisation de la consonne finale). À l'inverse, une ville comme Vannes connaît plusieurs variantes : les réponses vont de la méconnaissance à " Gwened », en passant par "Vann » prononcé à la française sans nasalisation (/van/), à « Woned », « Vened» ou « Gwenen ».

\section{Imaginaire linguistique}

En plus des communes à citer, il était également demandé aux informateurs :

1) ce qu'évoquaient pour eux les mots Bretagne, Basse- et HauteBretagne, s'ils savaient les distinguer, les définir et les dire en breton,

2) de dire la manière dont ils nomment l'endroit où ils habitent.

Ces informations davantage liées à l'imaginaire linguistique des informateurs peuvent permettre de mieux appréhender leurs préférences spatiales, les limites perçues, les sentiments d'appartenances à un territoire.

Sur les 31 informateurs, 10 (32\%) connaissent les mots « Breizh» et «Breizh-Isel ». Parmi ces 10 informateurs, une gradation va de la seule capacité à nommer "Breizh» (4 informateurs, soit 13\%) et «BreizhIsel » (1 informateur), à la capacité à qualifier les zones concernées, soit de manière approximative, soit de manière très précise ( 5 informateurs, soit 16\% du total) : "Berr ha krenn, Beiz-Isel zo Leon ha Breiz-Uhel zo Roazon », mais l'informateur précise ne pas les employer ; "Breizh, c'est la Bretagne entière, Breizh-Isel c'est l'ouest et Breizh-Uhel c'est l'est »; un autre, beaucoup plus précis, situe la frontière linguistique au nord entre Guingamp et Saint-Brieuc, deux autres situent Nantes en Bretagne et savent expliquer la différence linguistique entre les deux zones Breizh-Isel et Breizh-Uhel.

21 informateurs sur $31(68 \%)$ n'ont pas connaissance des mots «Breizh» et «Breizh-Isel » : beaucoup ne savent nommer ni la Bretagne, ni la Basse-Bretagne en breton; ils n'en connaissent pas les limites géographiques et ont une connaissance très floue de la géographie interne de ces zones et des différences linguistiques. L'une des informatrices se demande si on parle breton à Rennes ; un autre informateur, ne comprenant pas «Breizh», entend «Brest»; une autre ne sait quoi répondre à l'enquê- 
trice qui lui demande si Nantes est en Bretagne (à cette question idéologique, l'informatrice tente une réponse scolaire : «C'est en Normandie, je crois, non? ») ; un autre sent que le vannetais est bien différent de chez lui ; un autre trouve que le breton de Rennes et celui de Vannes se ressemblent; deux autres pensent que la Basse-Bretagne est située au sud (et qu'il y fait plus chaud) ; une perçoit Dinan et Dinard comme plus bretons que Rennes.

Certains enquêteurs ont tenté de pousser la réflexion en interrogeant les informateurs sur des zones plus locales comme le Bas- et le HautLéon, «Kreiz-Breizh », «Bro Bagan » et les pays comme le Trégor ou la Cornouaille. Ce petit examen poussé montre que, dans les zones léonardes couvertes, la distinction Bas- et Haut-Léon ne semble pas pertinente : soit elle n'est pas connue, soit elle suscite l'acquiescement par défaut (l'informateur de Plouider en déduit qu'il se situe plutôt en Bas-Léon car il sait que Saint-Pol-de-Léon « c'est le Haut-Léon »). «Kreiz-Breizh» ne semble pas être identifié, certains le renvoient loin («en tu-all»), d'autres le renvoient à la pratique d'autres personnes qu'eux ("Centre-Bretagne ? Peseurt mod 'lâront deja? Kreiz Breizh»). Le Pays Pagan n'est pas mieux identifié : "Lod a lavar emaomp e Bro Bagan, med ewidomp-ni n'omp ket Pagan » ou " je n'avais jamais entendu ça avant [la création de la troupe de théâtre Ar Vro Bagan] ». Quant aux pays, les informateurs de l'enquête en son état actuel sont potentiellement concernés par Léon/ Leon, Trégor/Treger et Cornouaille/Kerne. Hormis le Léon qui tire son épingle du jeu à la fois parce que des personnes s'y incluent et parce que d'autres le nomment en s'en excluant, «Treger » et « Kerne » restent des données pas ou peu utilisées spontanément, ni même sur sollicitations, et qui restent floues. À Botsorhel, les informatrices ne savent pas si elles sont en Trégor, mais pensent que oui. En revanche, elles identifient clairement le Léon et mobilisent à travers lui d'autres représentations que celles strictement territoriales : «Le Léon, c'est tout ce qui est mauvais. » À Plouigneau, Pommerit-le-Vicomte ou à Trégrom, on n'utilise pas le mot «Bro Dreger », à Belle-Isle-en-Terre, on ne sait pas si on est en Trégor ou en Cornouaille. " Ici, on dit qu'on est dans la Cornouaille, mais on n'en parlait pas », affirme un informateur d'Argol. À Mellionnec : « On est dans 'chais pas quoi. Pays Pourlet, à la limite, mais on est dedans quand même. Il y a quatre communes des Côtes-d'Armor dans le Pays Pourlet. On est dans la limite. On est pas dans le pays vannetais. Et j'en sais rien si c'est la Cornouaille. » À Trémargat, par contre, l'informateur répond « Me zo ur C'hernewad » et le marchand de chaussures de Morlaix, situé à la frontière des limites géographiques de trois évêchés et en contact avec des clients de plusieurs communes avoisinantes, connaît une gamme assez complète : il 
dit qu'il est «Tregeriad» et « Menez », et sait nommer Bro Dreger, Kerne, Leon, et leurs habitants Kernewiz et Leoniz.

D'autres repères géographiques sont fournis par les informateurs lors des entretiens. D'une part, la distinction entre les zones littorales et les zones intérieures : à Tréglonou, l'informatrice lance l'hypothèse que la limite entre le Haut- et le Bas-Léon recouvre peut-être la limite entre « le bord de mer et les terres », un informateur du Grouanec fait la distinction entre les gens de cette localité et ceux du bord de mer (autrement dit Lilia, partie littorale de la commune de Plouguerneau) et, lorsque l'enquêteur lui demande comment il dit « Le Havre » en breton, sa réponse témoigne de la spécificité des connaissances en la matière, restreinte à un corps de métier : « je sais qu'il y a un mot pour dire Le Havre, j'ai entendu les marins dire ça. » À Plouescat, « il y avait tud an Arvor ha tud ar Gorre et on avait des places différentes à l'église ». L'informateur de l'île de Batz signale aussi qu'il est de l'Armor. À Trémargat, loin de la mer, l'informatrice, rebondissant sur le terme «Kreiz Breizh» suggéré par l'enquêteur, répond : " 'Me zo deus kreiz an douar' a veze lâret » et parle de «An Argoad» par opposition à «Bro bord ar mor ».

Enfin, l'identification à un département est la plus remarquable. Environ un tiers des informateurs en parlent spontanément dans la conversation. C'est le plus souvent en français que sont cités les départements Finistère, Côtes-d'Armor, parfois Morbihan et Ille-et-Vilaine, et deux fois en breton pour le Finistère - Penn-ar-Bed : une informatrice précise qu'elle a lu ce nom sur le bulletin d'information du Conseil Général du Finistère mais qu'elle ne l'utilise pas, et l'autre affirme une partie de son identité et appartenance géographique : « Nous, on dirait qu'on est de Penn-ar-Bed. » Ce sentiment d'appartenance est parfois fortement marqué : «En Finistère on se sent plus breton que dans les autres départements », affirme l'informateur de Tréglonou. L'informateur de l'île de Groix, cherchant à expliquer pourquoi il ne sait pas dire en breton Carhaix, Morlaix et Rennes, se sert de deux types de limites, l'une départementale et l'autre linguistique : « Le Finistère, ça c'est pas chez nous », note-t-il aussi clairement que « Rennes, ça c'est gallo ».

\section{Interprétation}

« Comment ce siècle d'ouverture sur le monde se manifeste-t-il dans le savoir géographique vernaculaire breton? », nous demandions-nous en introduction. Les résultats de l'enquête, portant sur un échantillon d'un tiers des informateurs sur le total que nous espérons interroger d'ici la fin 
de l'enquête ${ }^{9}$, montre clairement le caractère endocentrique des représentations spatiales exprimées en breton. La combinaison d'une connaissance d'un espace proche de chez soi et restreint, avec une méconnaissance des espaces extérieurs plus ou moins éloignés est typique des représentations émanant de pratiques locales, de dénominations héritées, de savoirs nonenseignés et non pris en main par divers pouvoirs. Cela s'apparente à des emplois régis par des micro-consensus sociaux, locaux, n'excluant pas la variation dialectale d'une zone à une autre, épousant par là les traits caractéristiques des badumes ${ }^{10}$. À la question «Comment nommez-vous le lieu d'où vous venez? », plusieurs informateurs se rapportent à leur village (hameau) ou utilise l'expression «Bro + nom de la commune» (Bro Daole hag Henvig, Bro Wengamp, Bro Benac'h). Quant au flou, il est remarquable, non seulement par la méconnaissance des termes bretons pour dire «Bretagne » et « Basse-Bretagne », mais également par des hésitations à se situer dans un pays, par exemple en Pays Pagan : l'informateur de Plouguerneau dit n'avoir jamais entendu parler de cela avant, puis poursuit par « mais je crois qu'on est concerné aussi », tout en concluant «me 'gave drol Bro Bagan »; l'informateur de Plouescat pense ne pas être en Pays Pagan et croit que la limite se situe à Goulven.

Au flou géographique semble s'ajouter un flou plus ontologique qui révèle une absence de distinction nette entre l'espace, la langue et l'appartenance identitaire. La géographie mobilise ainsi d'autres types de représentations comme le montre l'exemple suivant dans la bouche de l'informateur de Plouguerneau qui indique que «Breizh, c'est notre langue parce qu'on dit 'O ! Breiz ma bro'».

Ce savoir vernaculaire, fortement basé sur une "géographie-badume ", est toutefois également nourri par des représentations véhiculées par l'Église, notamment en Léon. Cette institution coutumière semble y avoir imposé un niveau géographique et identitaire supérieur : «Leon» ou «Bro Leon» sont cités spontanément par plusieurs informateurs, alors que le Trégor et la Cornouaille semblent avoir été nettement moins reçus. Reste donc à mener l'enquête en pays vannetais pour savoir s'il s'agit d'une spécificité léonarde ou si la carte de la diagonale contestataire ${ }^{11}$ pourrait être

9. Une mise à jour de ces données sera effectuée lorsque l'ensemble des données sera rassemblé.

10. Pour une définition de ce concept, voir Jean LE DÛ \& Yves LE BerRe, «Parité et disparité : sphère publique et sphère privée de la parole », La Bretagne Linguistique, 10, Brest, CRBC-UBO, 1996, p. 7-25.

11. Pierre Flatrès, La Bretagne, Paris, PUF, 1986. 
superposable à celle que nous pourrions obtenir dans ce cas, ce qui montrerait l'impact plus fort de l'Église dans ces deux régions.

Quant aux institutions contemporaines à caractère plus normalisant, les enquêtés ne semblent pas s'approprier franchement les nouveaux découpages de l'espace qu'elles proposent. L'informateur de Plouguerneau suggère à l'enquêteur de se renseigner auprès de Goulc'han Kervella, directeur d'une troupe de théâtre en breton, pour savoir ce qu'est le « Pays Pagan ». L'informatrice de Dinéault s'appuie sur l'autorité du livre pour qualifier sa région : «Les Rouzig c'est les Bretons d'ici. Les Glazig c'est Quimper. Dans les bouquins on voit ça mais on ne disait pas ça »; tout comme l'informateur du Drennec qui parle de son pays comme du « pays du lin, pays des Julots », ou l'informateur de Plogonnec qui se base sur les éditions locales du quotidien régional pour fournir une réponse à l'enquêteur et évoque l'édition du sud-Cornouaille. La nouvelle signalétique bilingue promue et financée par les collectivités territoriales depuis quinze ans ne semblent pas avoir grand impact et laisse quelques informateurs perplexes : premièrement, il n'y a pas de réemploi des traductions proposées, et deuxièmement, certains mentionnent soit des difficultés à lire ce qui y est écrit («Aodoù-an-Arvor», en l'occurrence) ou le peu de différences entre le nom français et le nom breton («Plouguernével» en l'occurrence).

C'est l'enseignement qui semble l'autorité la plus efficace en la matière. Les deux exceptions déjà signalées, notamment pour leur propriété professionnelle, sont en effet dues au contact que les informateurs ont eu avec l'enseignement, soit en cours du soir, soit par l'intermédiaire d'un enseignant de breton qui a fourni une carte de la Bretagne en breton à l'un des informateurs. Ceux-ci sont ainsi capables de nommer des lieux de Basseet de Haute-Bretagne comme Fougères/Felger, la Loire-Atlantique/Liger Atlantel, et s'essaient même à l'invention de noms de communes lorsqu'ils n'en connaissent pas la forme vernaculaire, comme Gwinevez-ar-Faou (localement Plonevez-ar-Faou), sur le modèle de Gwinevez-Lokrist.

Pour autant, est-ce à dire que la mondialisation et l'ouverture des individus sur le monde tout au long du $\mathrm{XX}^{\mathrm{e}}$ siècle et en ce début du $\mathrm{XXI}^{\mathrm{e}}$ siècle n'a pas atteint la Basse-Bretagne ? Évidemment que non. La particularité de notre enquête par rapport à d'autres enquêtes du même genre sur la géographie mentale tient, certes, aux propriétés sociales de nos informateurs (leur âge, leur catégorie socioprofessionnelle, etc.) mais surtout dans la question du code employé. Si les informateurs ne savent pas dire Vannes, Bordeaux, Le Havre ou même Bretagne en breton, cela 
ne signifie pas qu'ils ne connaissent pas ces espaces et n'ont pas de mots pour le dire : «Me zo o chom ba Bretagne », dit par exemple l'informateur de Trégrom. Il est impossible de comprendre le sens sociolinguistique d'une telle phrase sans s'appuyer sur le concept de diglossie et la réalité diglossique des bretonnants enquêtés. Autre réponse du même ordre - diglossique par excellence - l'informateur d'Henvic dont les réponses semblent pourtant revêtir moins de caractère diglossique puisqu'il est capable de mentionner un échantillonnage plus large que la moyenne, enchaîne ses appartenances de la manière suivante : Bretagne, Breizh, France, Finistère, Bro Daole hag Henvig, alternant la nomination en français pour les appartenances larges et la nomination en breton pour l'appartenance locale, tout en y intercalant une traduction de Bretagne en breton. Mais c'est surtout la scolarisation en français dont on sent la plus grande influence sur l'appropriation de l'espace et des mots pour le dire. La réponse « Finistère, canton de Plouigneau, arrondissement de Morlaix, chef-lieu de Quimper, sous-préfecture de Morlaix » de l'informatrice de Botsorhel illustre parfaitement ce fort impact de l'institution scolaire sur les habitants de la Basse-Bretagne.

Finalement, la carte des lieux dans cet instantané pris au début du $\mathrm{XXI}^{\mathrm{e}}$ siècle ressemble étrangement à celle de la fin du XIX ${ }^{\mathrm{e}}$ siècle, à l'image de la géographie littéraire proposée par le prêtre Lan Inisan dans Emgann Kergidu ${ }^{12}$. Curieux résultat que cette machine qui, par la géographie, nous fait remonter le temps ${ }^{13}$.

12. Lan Inisan et Yves Le Berre, Emgann Kergidu - La Bataille de Kerguidu, Brest, CRBC, Coll. Bilingue Tal-ha-Tal, 2015 ; et Yves Le Berre, Emgann Kergidu de Lan Inisan : un homme, une œuvre, un terroir, thèse, Université de Brest, 1978.

13. Ce qui n'est pas sans rappeler les hypothèses géolinguistiques issues de l'analyse des l'ALF. Cf. Guylaine Brun-Trigaud, Yves Le Berre, Jean Le Dû, Lectures de l'Atlas Linguistique de la France de Gilliéron et Edmont. Du temps dans l'espace, Paris, Comité des travaux historiques et scientifiques, 2005. 
Annexe : Carte des cantons couverts entre 2011 et 2014

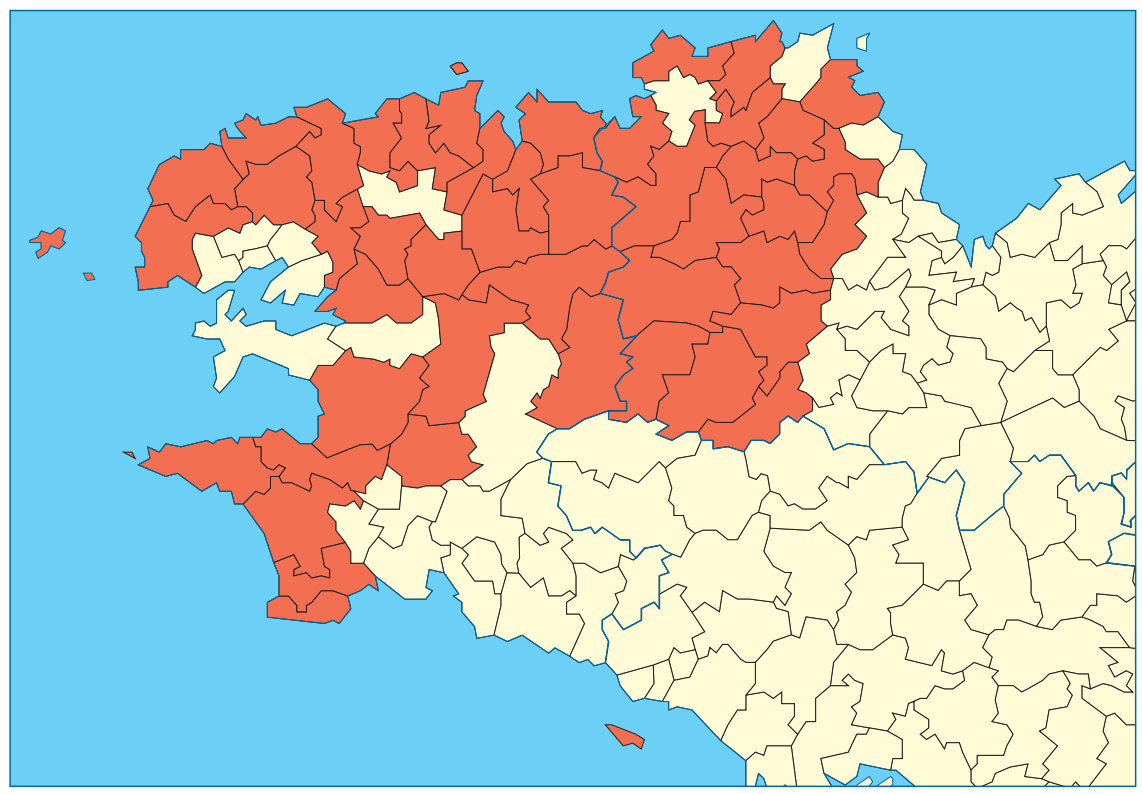


Actualités

de la recherche 